OPEN

SUBJECT AREAS:

FERROELECTRICS AND MULTIFERROICS

APPLIED PHYSICS

Received

29 October 2014

Accepted

12 January 2015

Published

5 February 2015

Correspondence and requests for materials should be addressed to Y.S. (youngsun@iphy. ac.cn)

\section{Nonvolatile electric-field control of magnetization in a Y-type hexaferrite}

\author{
Shipeng Shen, Yisheng Chai \& Young Sun
}

Beijing National Laboratory for Condensed Matter Physics, Institute of Physics, Chinese Academy of Sciences, Beijing 100190, China.

The magnetoelectric effects in multiferroic materials enable the mutual control of electric polarization by a magnetic field and magnetization by an electric field. Nonvolatile electric-field control of magnetization is extremely important for information storage applications, but has been rarely realized in single-phase multiferroic materials. Here we demonstrate the prominent direct and converse magnetoelectric effects in the Y-type hexaferrite $\mathrm{BaSrCoZnFe}{ }_{11} \mathrm{AlO}_{22}$ single crystal. The electric polarization due to conical magnetic structure can be totally reversed by a small magnetic field, giving rise to large magnetoelectric coefficients of 6000 and $4000 \mathrm{ps} / \mathrm{m}$ at 100 and $200 \mathrm{~K}$, respectively. The $a b$-plane magnetization can be controlled by electric fields with a large hysteresis, leading to nonvolatile change of magnetization. In addition, the reversal of magnetization by electric fields is also realized at $200 \mathrm{~K}$. These diverse magnetoelectric effects with large coefficients highlight the promise of hexaferrites as potential multiferroic materials.

lectric-field (E) control of magnetism has aroused intense interests due to its potential to develop new spintronic and electronic devices ${ }^{1-3}$. Many schemes have been attempted in the past decade to achieve this goal. In heterostructures and thin films, magnetization can be electrically modified via strain from piezoelectric substrates ${ }^{4-9}$; via exchange bias from antiferromagnetic-ferroelectric $\left(\mathrm{BiFeO}_{3}\right)^{10-12}$ or magnetoelectric $\left(\mathrm{Cr}_{2} \mathrm{O}_{3}\right)$ substrates ${ }^{13-14}$ etc. For the case of single-phase materials, magnetoelectrics and magnetoelectic (ME) multiferroics are top candidates to achieve the direct $E$ control of magnetizations $(M)$ because of its intrinsic ME coupling ${ }^{15-19}$. However, such a converse ME effect usually requires a large external magnetic field $(\mathrm{H})$ or low temperature, which is not practical for device application. Furthermore, nonvolatile $E$ control of magnetization, which is important for information storage, poses more severe challenges for ME multiferroics.

Hexaferrites with conical magnetic structures are promising multiferroic materials which show direct ME effect (the manipulation of polarization $P$ by $H$ ) up to room temperature ${ }^{20-24}$. The converse ME effect was also observed in several hexaferrites ${ }^{25-28}$. For instance, $E$ control of $M$ was realized in $\mathrm{Ba}_{0.5} \mathrm{Sr}_{1.5} \mathrm{Zn}_{2}\left(\mathrm{Fe}_{0.92} \mathrm{Al}_{0.08}\right)_{12} \mathrm{O}_{22}$ single crystal below $170 \mathrm{~K}$. Even at room temperature, converse ME effects were still observed in Y- and Z-type hexaferrites ceramics. Nevertheless, the reported converse ME effects are usually volatile - after removing $E$ field, $M$ restores the initial state. Although nonvolatile $E$ control of $M$ was recently reported in Y-type hexaferrite ceramics $^{27}$, the hysteresis of the $M-E$ curve is very small, with a tiny remanent magnetization in zero $E$ field, which is unsuitable for nonvolatile applications. In this work, we performed a systematic study on both the direct and converse $\mathrm{ME}$ effects in the Y-type hexaferrite $\mathrm{BaSrCoZnFe}_{11} \mathrm{AlO}_{22}$ single crystal. The results demonstrate pronounced ME effects with the direct ME coefficients of 6000 and 4000 ps/m at 100 and $200 \mathrm{~K}$, respectively. Moreover, the converse ME effect exhibits a large hysteresis in the $M-E$ loop which enables a clear nonvolatile $E$ control of $M$.

\section{Results}

Characterization of the Y-type hexaferrite $\mathbf{B a S r C o Z n F e}_{11} \mathbf{A l O}_{22}$. As shown in Fig. 1a, the Y-type hexaferrite has a stacked layer structure with the space group $R-3 \mathrm{~m}$. Our prepared single crystals of $\mathrm{BaSrCoZnFe}_{11} \mathrm{AlO}_{22} \mathrm{were}$ characterized by single crystal $\mathrm{X}$-ray diffraction (XRD), and the room-temperature XRD pattern (Fig. 1b) suggests that our specimen belongs to Y-type hexaferrite with $c=4.32 \mathrm{~nm}$.

Fig. 1c shows the temperature ( $T$ ) dependent magnetization curve with $H=100$ Oe along the [100] direction. Before the measurement, an external magnetic field $H=10 \mathrm{kOe}$ was first applied at $10 \mathrm{~K}$ to induce commensurate transverse cone state ${ }^{29}$, which is an intermediate ferroelectric (FE) phase according to the spin-current (KNB) or inverse Dzyaloshinskii-Moriya (DM) interaction model $\mathrm{s}^{30,31}$; then $H$ was ramped down to 100 Oe. The commensurate transverse cone would be kept under $H=100$ Oe. Two critical temperatures $T_{1}$ and $T_{2}$ are 

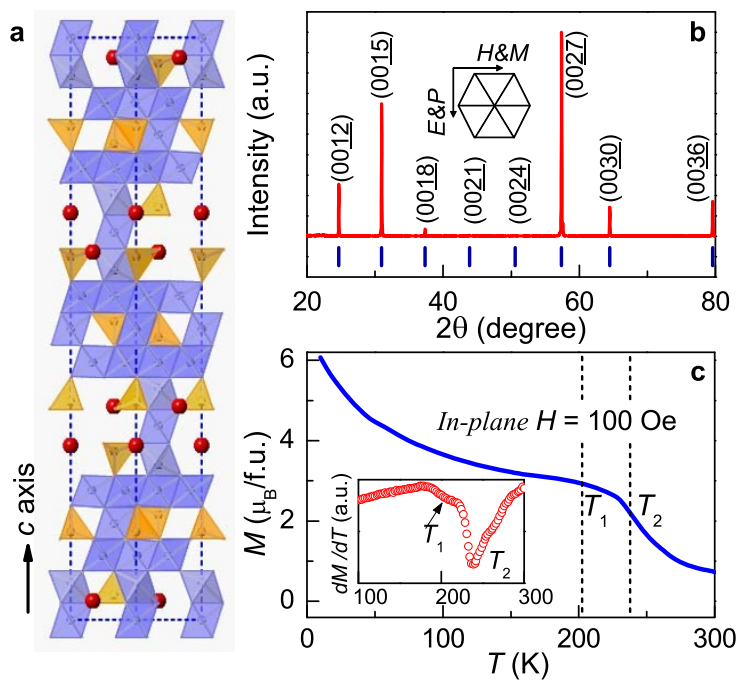

Figure $1 \mid$ Characterization of the Y-type hexaferrite $\mathrm{BaSrCoZnFe}_{11} \mathrm{AlO}_{22}$. (a) The schematic crystal structure of Y-type hexaferrite. (b) The X-ray diffraction pattern of the Y-type hexaferrite single crystal sample along [001] direction. Inset of panel (b) shows the schematic experimental configuration. (c) Temperature dependent magnetization with $H=100$ Oe along [100] axis. Before the measurements, $H=10 \mathrm{kOe}$ was applied at $10 \mathrm{~K}$ to induce a metastable commensurate transverse cone state, then $H$ was ramped down to 100 Oe. The Inset shows the derivative $\mathrm{d} M / \mathrm{d} T$ as a function of temperature.

observed in the $M-T$ and $\mathrm{d} M / \mathrm{d} T$ curves (Fig. 1c). At $T_{1} \sim 200 \mathrm{~K}$ the magnetization starts to drop more abruptly, suggesting that the commensurate transverse cone is no longer stable near zero magnetic field. The magnetodielelectric and magnetoelectric current measurements, as discussed in next section, also suggest that the commensurate transverse cone is likely to change to the longitudinal cone above $T_{1}$ due to the change of magnetic anisotropy. With further increasing temperature, there is another critical temperature $T_{2}$ and it is likely to be the transition to proper screw due to its easy in-plane anisotropy at higher temperatures ${ }^{32}$.

Magnetic field control of electric polarization. To check the nature of magnetic-order-induced FE phase, we measured the in-plane $(H / /$ [100] and $E / /$ [120]) magnetodielectric properties at different temperatures. Fig. 2a shows the relative change of dielectric constant, $\Delta \varepsilon(H) / \varepsilon(50 \mathrm{kOe})=[\varepsilon(H)-\varepsilon(50 \mathrm{kOe})] / \varepsilon(50 \mathrm{kOe})$, at selected temperatures. At high fields, there are broad peaks at all temperatures, corresponding to the transitions from $\mathrm{PE}$ to $\mathrm{FE}$ or $\mathrm{FE}$ to $\mathrm{PE}$ phase. Fig. $2 \mathrm{~b}$ shows the detailed magnetodielectric behaviors near zero field. Below $200 \mathrm{~K}$, only one single dielectric constant peak appears at low magnetic field, indicating the switching of FE domain, which is also confirmed by the $P-H$ curves in Fig. 2d. At $200 \mathrm{~K}$, a slight shoulder feature starts appearing at finite $H$, signaling a new phase coexists with FE phase near zero field. This new phase is none other than PE phase mentioned above. With further rising temperature, the intensity of the shoulders increase gradually and the height of zero-field single peak decreases simultaneously. At $300 \mathrm{~K}$, the zero-field single peak completely disappears and is replaced by the double peaks around $H$ $= \pm 2 \mathrm{kOe}$, which marks FE $-\mathrm{PE}-\mathrm{FE}$ double phase transitions. All these magnetodielectric behaviors are in accordance with the $M-T$ curve (Fig. 1c). Based on the magnetodielectric data, we obtain the magnetoelectric phase diagram shown in Fig. $2 \mathrm{c}$. Below $T_{1}$, only transverse cone (FE phase) exists around zero fields and the reversal of electric polarization by magnetic field can be attributed to direct in-plane reversal of the transverse cone state. Above $T_{1}$, the PE phase would emerge and coexist with the FE phase near zero fields. With temperature further rising, the PE phase gradually dominates near zero fields at high temperatures.

We also measured the magnetoelectric current $\left(J_{\mathrm{ME}}\right)$ below $T_{1}$ and integrated it by time to obtain electric polarization $(P)$, as displayed in Fig. $2 \mathrm{~d}$. The $P$ can be reversed by a small $H$. The ME coefficients $\left(\alpha_{h}\right.$ $=\partial P / \partial H)$ calculated by the magnetoelectric current reach a maximum of $\sim 6000 \mathrm{ps} / \mathrm{m}$ at 100 and $150 \mathrm{~K}$, and $\sim 4000 \mathrm{ps} / \mathrm{m}$ at $200 \mathrm{~K}$. Above $200 \mathrm{~K}$, because of the low resistivity of our crystal at high temperature, we are not able to pole the sample to obtain reliable
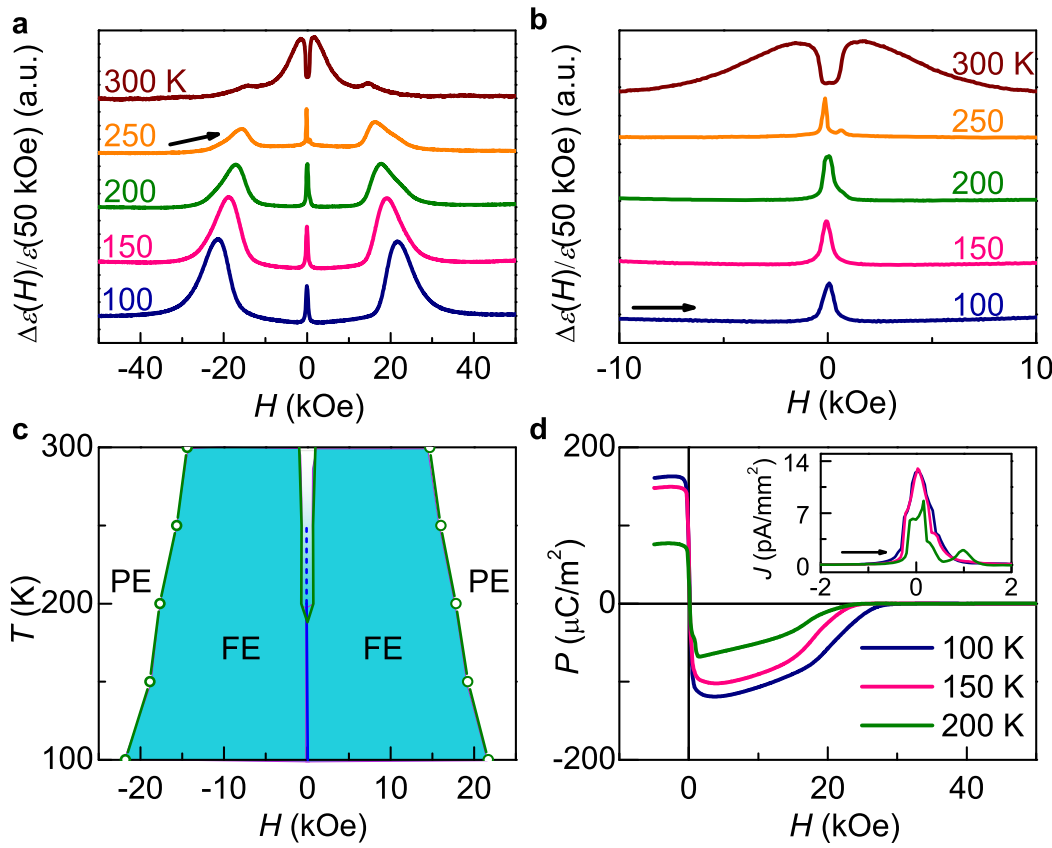

Figure $2 \mid$ Magnetic field control of electric polarization. (a) The magnetodielectric ratio $\Delta \varepsilon(H) / \varepsilon(50 \mathrm{kOe})=[\varepsilon(H)-\varepsilon(5 \mathrm{kOe})] / \varepsilon(5 \mathrm{kOe})$ at selected temperatures. (b) The details of the magnetodielectric behavior around zero field. (c) The magnetoelectric phase diagram of $\mathrm{BaSrCoZnFe}_{11} \mathrm{AlO}_{22}$. (d) Magnetic field reversal of in-plane electric polarization at 100, 150, and $200 \mathrm{~K}$. The inset shows the magnetoelectric current near zero magnetic field. 
ME current. The inset of Fig. $2 \mathrm{~d}$ shows the $J_{\mathrm{ME}}$ around zero magnetic fields. At $200 \mathrm{~K}$, besides the current peak at zero field like those at $100 \mathrm{~K}$ and $150 \mathrm{~K}$,there is a small current peak around $H=1 \mathrm{kOe}$ corresponding to the magnetodielectric shoulder. Therefore, we can attribute the weak current peak to the transition of $\mathrm{PE}$ to FE, which suggests chirality is unchanged during the transverse cone - longitudinal cone - transverse cone transitions, similar to that reported in $\mathrm{Ba}_{2} \mathrm{Mg}_{2} \mathrm{Fe}_{12} \mathrm{O}_{22}$ (Ref. 21). Moreover, the little current peak also verifies the existence of PE phase near zero magnetic field.

Nonvolatile electric control of magnetization. We then focus on the converse $\mathrm{ME}$ effect in the $\mathrm{BaSrCoZnFe}{ }_{11} \mathrm{AlO}_{22}$ single crystal sample. For multiferroics, there usually exist four kinds of compound domains signified by magnetic and electric order parameters $\pm M$ and $\pm P^{33}$. In Y-type hexaferrite, the in-plane $P$ is caused by long range transverse conical magnetic order with wave vector along $c$ axis, which is in accordance with the spin-current (KNB) model. Therefore, the directions of $P$ and $M$ of compound domain are totally dependent on the electric field $E$ and magnetic field $H$ during poling process. To achieve the converse ME effect as large as possible, the converse $\mathrm{ME}$ effects were measured after a $\mathrm{ME}$ poling procedure in which $H$ was decreased from $50 \mathrm{kOe}$ to $5 \mathrm{kOe}$ under application of $E=500 \mathrm{kV} / \mathrm{m}$ and then the $E$ field was turned off. After that, $H$ was set to zero or a low field for the converse ME effect measurement.

Fig. 3a shows the $E$ dependence of $M$ along [100] direction at $100 \mathrm{~K}$. The maximum $E$ for the converse ME effect measurement is limited under $1 \mathrm{MV} / \mathrm{m}$ for safety reason. The substantial hysteresis between the data obtained during increasing and decreasing $E$ scan produces a well-defined $M-E$ hysteresis loop. To estimate the converse ME coefficients in the $M-E$ hysteresis case, the magnetizations of the increasing and decreasing $E$-scan data were averaged. A quad-
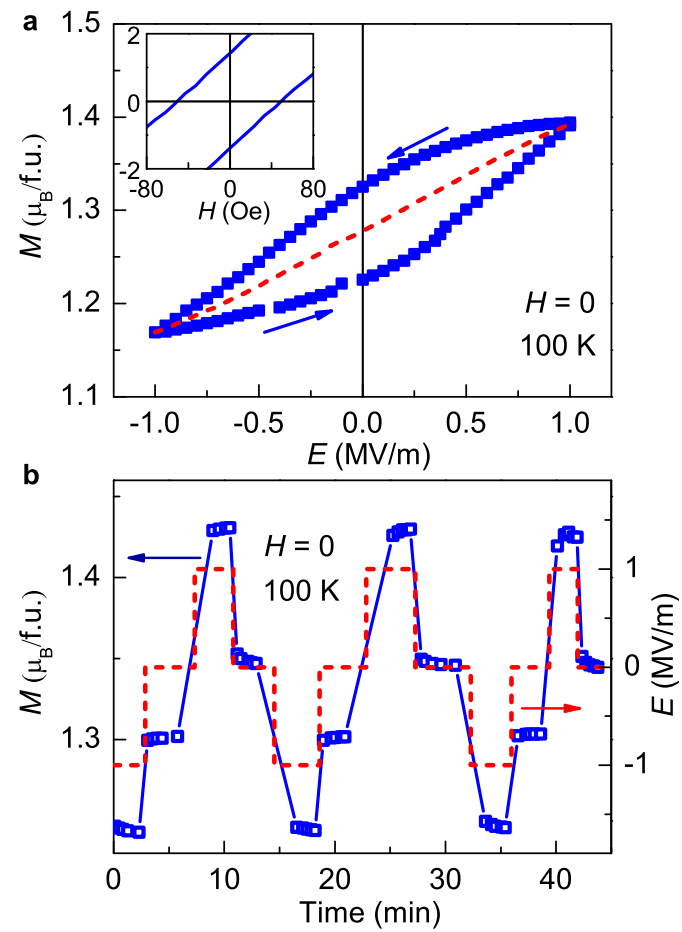

Figure 3 Nonvolatile electric control of magnetization.

(a) Magnetization as a function of electric field at $100 \mathrm{~K}$ showing the $M-$ $E$ hysteresis loop. The inset shows the $M-H$ hysteresis at low magnetic fields. (b) Four magnetization levels controlled by applying electric field in a repeated sequence of $-1 \mathrm{MV} / \mathrm{m} \rightarrow 0 \rightarrow 1 \mathrm{MV} / \mathrm{m} \rightarrow 0$. No bias magnetic field is needed. ratic function: $M(E)-M(0)=\alpha_{e} E+\frac{1}{2} \gamma E^{2}$ is used to approximate the $E$ dependence of $M$, where it includes linear and quadratic $E$ terms $^{25}$. The linear coefficient $\alpha_{e}=3100 \mathrm{ps} / \mathrm{m}$ and quadratic coefficient $\gamma=80 \mathrm{ps} / \mathrm{MV}$ were obtained, indicating the converse ME effect at $100 \mathrm{~K}$ is mainly dominated by the linear term. The smaller difference between the values of $\alpha_{e}$ and $\alpha_{h}$ in single crystal than ceramic samples ${ }^{27}$ suggests that the contribution from trapped space charges is effectively suppressed.

The large $M-E$ hysteresis loop at $100 \mathrm{~K}$ indicates its potential for nonvolatile magnetization controlled by $E$, shown in Fig. 3b. After the poling procedure mentioned above, we measured $M$ with applying $E$ field in a sequence: $-1 \mathrm{MV} / \mathrm{m}-0-1 \mathrm{MV} / \mathrm{m}-0$ without a magnetic field bias. When applying a negative $E$, the magnetization is reduced owing to the clamped $M \& P$ orders. In contrast, the positive $E$ leads to an increase in $M$. It clearly displays that two magnetization values at $E=0$ are distinctively different after remove $E=1 \mathrm{MV} / \mathrm{m}$ and $E=-1 \mathrm{MV} / \mathrm{m}$, due to its large $M-E$ hysteresis loop shown in Fig. 3a. Moreover, no obvious damping of magnetization was observed after several periods, though we did not apply large enough $E$ to make the magnetization saturated.

The reversal of magnetization by electric field. Next, we performed similar measurements at $200 \mathrm{~K}$. Fig. 4 a displays the $M-E$ curve showing a large hysteresis at $200 \mathrm{~K}$ with external $H=-10 \mathrm{Oe}$ along [100] direction. Compared with that at $100 \mathrm{~K}$, the $M-E$ curve at $200 \mathrm{~K}$ is more asymmetric and $M$ almost remains constant with decreasing negative $E$-scan. With the same fitting method taken at $100 \mathrm{~K}$, we got $\alpha_{e}=1100 \mathrm{ps} / \mathrm{m}$ and $\gamma=535 \mathrm{ps} /$ $\mathrm{MV}$ at $200 \mathrm{~K}$. Such a large quadratic coefficient $\gamma$ is likely to be the reason for the asymmetric shape of the $M-E$ curve at $200 \mathrm{~K}$. This quadratic converse ME effect is possibly introduced by the new PE phase around zero magnetic field, as our magnetic and magnetodielectric measurements have suggested the existence of mixed phases at this temperature and the transverse cone is
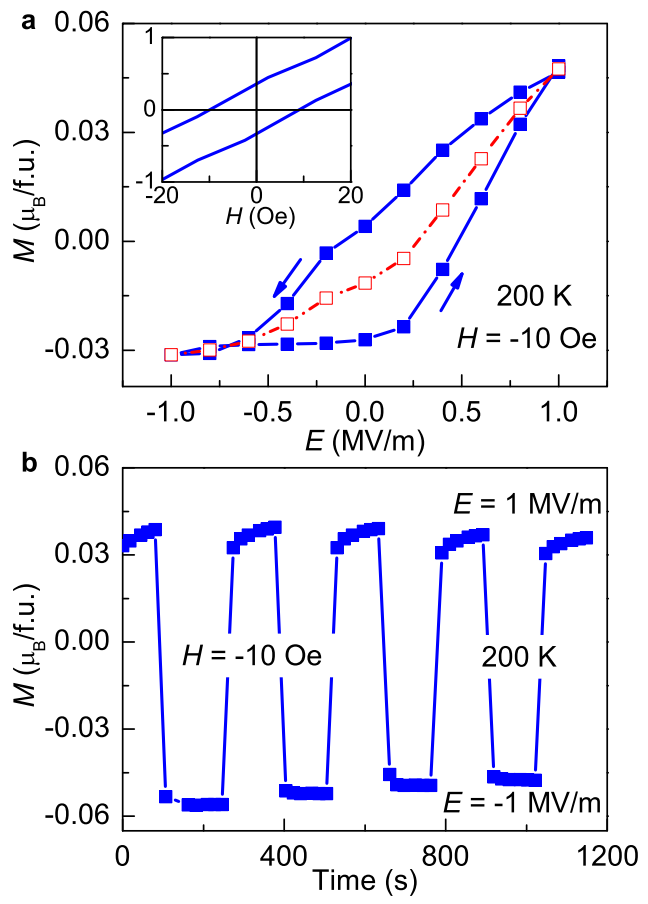

Figure $4 \mid$ The reversal of magnetization by electric field.

(a) Magnetization as a function of electric field measured with a bias magnetic field $H=-10$ Oe at $200 \mathrm{~K}$. The inset shows the $M-H$ hysteresis loop at low magnetic fields. (b) Electric-field reversal of magnetization under a small bias magnetic field $H=-10$ Oe. 
responsible for the linear converse $\mathrm{ME}$ effect, which has been evidenced by the results at $100 \mathrm{~K}$. the substantial hysteresis at $200 \mathrm{~K}$ suggests its potential for nonvolatile magnetic states controlled by $E$, just like that at $100 \mathrm{~K}$. A distinguished feature at $200 \mathrm{~K}$ is the reversal of $M$ by $E$ field. Fig. $4 \mathrm{~b}$ demonstrates the repeated reversal of $M$ by switching the polarity of electric field ( $E$ $= \pm 1 \mathrm{MV} / \mathrm{m}$ ) under a small bias $H=-10$ Oe at $200 \mathrm{~K}$. The positive $E$ causes an increase in $M$, while the negative $E$ leads to polarity reversal of $M$.

\section{Discussion}

The electric polarization in Y-type hexaferrite can be well explained by the spin-current model $\boldsymbol{P} \propto \boldsymbol{k}_{0} \times\left(\boldsymbol{S}_{i} \times \boldsymbol{S}_{j}\right)$, where $\boldsymbol{S}_{\boldsymbol{i}}$ and $\boldsymbol{S}_{j}$ denote the neighbor spins at site $i$ and $j, \boldsymbol{k}_{\boldsymbol{0}} / /$ [001] is the magnetic propagation vector. According to this model, the screw or longitudinal conical spin configurations cannot generate $P$ due to spin chirality $\Sigma\left(S_{i}\right.$ $\left.\times S_{j}\right) / / \boldsymbol{k}_{0}$. However, the transverse cone is a FE phase. Below $200 \mathrm{~K}$, the transverse cone (FE) still persists after we decrease $H$ from high field to zero, which is responsible for the direct reversal of $P$ by $H$ and the linear converse ME effect. In benefit of the large converse ME susceptibility $\left(\alpha_{e}=3100 \mathrm{ps} / \mathrm{m}\right)$ and substantial $M-E$ hysteresis loop, we achieve nonvolatile distinguished change of $M$ by $E$, that is to say, the magnetizations differ even after removing $+E$ and $-E$. This result provides the potential for future nonvolatile magnetic memory device controlled by $E$.

Due to the clamped $M \& P$ orders in multiferroic hexaferrites, there are only two kinds of compound domains: $+M /+P$ and $-M /-P$ since $+E$ and $+H$ are applied during poling process. The manipulation of $M$ by $E$ can be thought as the result of the compound domain wall movement. Like the hysteresis loop in the $M-H$ loop, the hysteresis characteristics for $M-E$ curves appear due to resistance of domain wall movement. The relatively large coercive field $\left(H_{c}\right.$ $=50 \mathrm{Oe}$ ) in the $M-H$ loop is probably responsible for the substantial $M-E$ hysteresis loop, which is the prerequisite for nonvolatile magnetization controlled by $E$.

Different from the case at $100 \mathrm{~K}$ where the linear converse ME effect dominates, the contribution of quadratic converse ME effect is no longer negligible at $200 \mathrm{~K}$. Magnetodielectric and $J_{\mathrm{ME}}$ measurements at $200 \mathrm{~K}$ suggest that $\mathrm{PE}$ and FE phase coexist at zero field, that is to say, besides the transverse cone phase, there is a longitudinal cone or proper screw phase when we ramp from high- $H$ down to zero. Thus, it could be the existence of the PE phase (proper screw or longitudinal cone) responsible for the quadratic converse ME effect.

In summary, we have demonstrated pronounced ME effects in the Y-type hexaferrite $\mathrm{BaSrCoZnFe}_{11} \mathrm{AlO}_{22}$ single crystal at different temperatures. Its direct $\mathrm{ME}$ susceptibility can reach as high as $6000 \mathrm{ps} / \mathrm{m}$ at $100 \mathrm{~K}$, and $4000 \mathrm{ps} / \mathrm{m}$ at $200 \mathrm{~K}$. In benefit of the large $M-E$ hysteresis loop as well as the large converse ME coefficients ( 3100 and $1100 \mathrm{ps} / \mathrm{m}$ at 100 and $200 \mathrm{~K}$, respectively) in single crystals, nonvolatile $E$ control of magnetization can be realized. In addition, reversal of magnetization by $E$ field was also demonstrated at $200 \mathrm{~K}$. These excellent properties promise its potential for the future magnetoelectric functional devices. Further tailoring of the composition of Y-type hexaferrites may eventually realize these diverse ME effects at room temperature.

\section{Methods}

Sample preparation. Single-crystal samples of the Y-type hexaferrite $\mathrm{BaSrCoZnFe}_{11} \mathrm{AlO}_{22}$ were prepared by $\mathrm{Na}_{2} \mathrm{O}-\mathrm{Fe}_{2} \mathrm{O}_{3}$ flux method in air as described elsewhere ${ }^{34}$. The as-grown samples were annealed at $900^{\circ} \mathrm{C}$ in $\mathrm{O}_{2}$ atmosphere to enhance the resistivity ${ }^{35}$

Electric and magnetic measurements. For electrical measurements, the crystals were cut into thin plates with the widest faces perpendicular to the [120] direction in the hexagonal setting and then were painted with sliver paste on the widest faces. The $J_{M E}$ and dielectric constant were measured by an electrometer (Keithley 6517B) and a LCR meter (Aglient, 4980A), respectively, in a Cryogen-free Superconducting Magnet System (Oxford Instruments, TeslatronPT). The converse ME effects were measured by using a magnetometer with a homemade sample holder (MPMS, Quantum Design). Before $J_{\mathrm{ME}}$ measurements, we need to carry out the following steps to pole our sample: (1) $E=500 \mathrm{kV} / \mathrm{m}$ was applied at $H=50 \mathrm{kOe}$ along [100] direction where sample was in a high-field PE phase; (2) ramped down the $H$ to $5 \mathrm{kOe}$, where the sample was driven to the intermediate field FE phase, then withdrew electric field and shortened two electrodes for 30 mins to release free charges; (3) swept magnetic field with $25 \mathrm{Oe} / \mathrm{s}$ to $-50 \mathrm{kOe}$ and measured the $J_{\mathrm{ME}}$.

1. Spaldin, N. A. \& Fiebig, M. The renaissance of magnetoelectric multiferroics. Science 309, 391-392 (2005).

2. Eerenstein, W., Mathur, N. D. \& Scott, J. F. Multiferroic and magnetoelectric magterials. Nature 442, 759-765 (2006).

3. Spaldin, N. A., Cheong, S. W. \& Ramesh, R. Multferroics: Past, present, and future. Phys. Today 63, No. 10, 38 (2010).

4. Eerenstein, W., Wiora, M., Prieto, J. L., Scott, J. F. \& Mathur, N. D. Giant sharp and persistent converse magnetoelectric effects in multiferroic epitaxial heterostructures. Nat. Mater. 6, 348-351 (2007).

5. Sahoo, S. et al. Ferroelectric control of magnetism in $\mathrm{BaTiO}_{3} / \mathrm{Fe}$ heterostructures via interface strain coupling. Phys. Rev. B 76, 092108 (2007).

6. Thiele, C., Dörr, K., Bilani, O., Rödel, J. \& Schultz, L. Influence of strain on the magnetization and magnetoelectric effect in $\mathrm{La}_{0.7} \mathrm{~A}_{0.3} \mathrm{MnO}_{3} / \mathrm{PMN}-\mathrm{PT}(001)(\mathrm{A}=$ Sr, Ca). Phys. Rev. B 75, 054408 (2007).

7. Weiler, M. et al. Voltage controlled inversion of magnetic anisotropy in a ferromagnetic thin film at room temperature. New J. Phys. 11, 013021 (2009).

8. Ghidini, M. et al. Non-volatile electrically driven repeatable magnetization reversal with no applied magnetic field. Nature comm. 4, 1453 (2012).

9. Zhang, S. et al. Electric-Field control of nonvolatile magnetization in $\mathrm{Co}_{40} \mathrm{Fe}_{40} \mathrm{~B}_{20}$ $\mathrm{Pb}\left(\mathrm{Mg}_{1 / 3} \mathrm{Nb}_{2 / 3}\right)_{0.7} \mathrm{Ti}_{0.3} \mathrm{O}_{3}$ structure at room temperature. Phys. Rev. Lett. 108, 137203 (2012).

10. Zhao, T. et al. Electrical control of antiferromagnetic domains in multiferroic $\mathrm{BiFeO}_{3}$ films at room temperature. Nat. Mater. 5, 823-829 (2006).

11. Lebeugle, D. et al. Electric field switching of the magnetic anisotropy of a ferromagnetic layer exchange coupled to the multiferroic compound $\mathrm{BiFeO}_{3}$. Phys Rev. Lett. 103, 257601 (2009)

12. Chu, Y. H. et al. Electric-field control of local ferromagnetism using a magnetoelectric multiferroic. Nat. Mater. 7, 478-482 (2008).

13. Wu, N. et al. Imaging and control of surface magnetization domains in a magnetoelectric antiferromagnet. Phys. Rev. Lett. 106, 087202 (2011).

14. He, X. et al. Robust isothermal electric control of exchange bias at room temperature. Nat. Mater. 9, 579-585 (2010).

15. Iyama, A. \& Kimura, T. Magnetoelectric hysteresis loop in $\mathrm{Cr}_{2} \mathrm{O}_{3}$ at room temperature. Phys. Rev. B 87, 180408(R) (2013).

16. Lottermoser, T. et al. Magnetic phase control by an electric field. Nature 430 541-544 (2004)

17. Tokunaga, Y., Taguchi, Y., Arima, T. \& Tokura, Y. Electric-field-induced generation and reversal of ferromagnetic moment in ferrites. Nature Phys. 8, 838-844 (2012).

18. Tian, Y. et al. Electric control of magnetism in a multiferroic metal-organic framework. Phys. Status Solidi RRL 8, 91-94 (2014).

19. Tian, Y. et al. Cross coupling between electric and magnetic orders in a multiferroic metal-organic framework. Sci. Rep. 4, 6062 (2014).

20. Kimura, T., Lawes, G. \& Ramirez, A. P. Electric polarization rotation in a hexaferrite with long-wavelength magnetic structures. Phys. Rev. Lett. 94, 137201 (2005).

21. Ishiwata, S. et al. Low-magnetic-field control of electric polarization vector in a helimagnet. Science 319, 1643-1646 (2008).

22. Chun, S. H. et al. Realization of giant magnetoelectricity in helimagnets. Phys. Rev. Lett. 104, 037204 (2010).

23. Wang, F., Zou, T., Yan, L. Q., Liu, Y. \& Sun, Y. low magnetic field reversal of electric polarization in a Y-type hexaferrite. Appl. Phys. Lett. 100, 122901 (2012).

24. Kitagawa, Y. et al. Low-field magnetoelectric effect at room temperature. Nature Mater. 9, 797-802 (2010)

25. Chun, S. H. et al. Electric field control of nonvolatile four-state magnetization at room temperature. Phys. Rev. Lett. 108, 177201 (2012).

26. Chai, Y. S. et al. Electric control of large magnetization reversal in a helimagnet. Nature Comm. 5, 4208 (2014).

27. Hirose, S., Haruki, K., Ando, A. \& Kimura, T. Mutual control of magnetization and electric polarization by electric and magnetic field at room temperature in $\mathrm{Y}$ type $\mathrm{BaSrCo}_{2-\mathrm{x}} \mathrm{Zn}_{\mathrm{x}} \mathrm{Fe}_{11} \mathrm{AlO}_{22}$ ceramics. Appl. Phys. Lett. 104, 022907 (2014).

28. Okumura, K. et al. Multilevel magnetization switching by electric field in c-axis oriented polycrystalline Z-type hexaferrite. Appl. Phys. Lett. 103, 032906 (2013)

29. Shen, S. P. et al. Magnetic field reversal of electric polarization and magnetoelectric phase diagram of the hexaferrite $\mathrm{Ba}_{1.3} \mathrm{Sr}_{0.7} \mathrm{Co}_{0.9} \mathrm{Zn}_{1.1} \mathrm{Fe}_{10.8} \mathrm{Al}_{1.2} \mathrm{O}_{22}$. Appl. Phys. Lett. 104, 032905 (2014).

30. Katsura, H., Nagaosa, N. \& Balatsky, A. V. Spin current and magnetoelectric effect in noncollinear magnets. Phys. Rev. Lett. 95, 057205 (2005).

31. Sergienko, I. A. \& Dagotto, E. Role of the Dzyaloshinskii-Moriya interaction in multiferroic perovskites. Phys. Rev. B 73, 094434 (2006).

32. Smit, J. \& Wijn, H. P. J. Ferrites (Phillips Technical Library, Eindhoven, 1959).

33. Tokunaga, Y. et al. Composite domain walls in a multiferroic perovskite ferrite Nature Mater. 8, 558-562 (2009). 
34. Momozawa, N., Mita, M. \& Takei, H. Single crystal growth of $\left(\mathrm{Ba}_{1-\mathrm{x}} \mathrm{Sr}_{\mathrm{x}}\right)_{2} \mathrm{Zn}_{2} \mathrm{Fe}_{12} \mathrm{O}_{22}$ from high temperature solution. J. Cryst. Growth 83, 403-409 (1987).

35. Chai, Y. S. et al. Low-magnetic-field control of dielectric constant at room temperature realized in $\mathrm{Ba}_{0.5} \mathrm{Sr}_{1.5} \mathrm{Zn}_{2} \mathrm{Fe}_{12} \mathrm{O}_{22}$. New J. Phys. 11, 073030 (2009).

\section{Acknowledgments}

This work was supported by the National Key Basic Research Program of China under Grant No. 2011CB921801, the Natural Science Foundation of China under Grants Nos 11227405 and 11374347, and the Strategic Priority Research Program of the Chinese Academy of Sciences under Grant No. XDB07030200.

\section{Author contributions}

Y.S. and S.S conceived the project. S.S prepared the samples and performed the experiments. Y.C. helped in experiments and analysis of the data. All authors contributed to writing the paper.

\section{Additional information}

Competing financial interests: The authors declare no competing financial interests.

How to cite this article: Shen, S., Chai, Y. \& Sun, Y. Nonvolatile electric-field control of magnetization in a Y-type hexaferrite. Sci. Rep. 5, 8254; DOI:10.1038/srep08254 (2015).

This work is licensed under a Creative Commons Attribution 4.0 International License. The images or other third party material in this article are included in the article's Creative Commons license, unless indicated otherwise in the credit line; if the material is not included under the Creative Commons license, users will need to obtain permission from the license holder in order to reproduce the material. To view a copy of this license, visit http://creativecommons.org/licenses/by/4.0/ 